\title{
Research on Building Customized Furniture Product Model and Assembly Sequence
}

\author{
${ }^{1}$ Qi Yue and ${ }^{2}$ Yang Sun \\ College of Information and Computer Engineering \\ Northeast Forestry University Harbin Heilongjiang, 150040, China \\ ${ }^{1}$ E-mail:491558028@qq.com, ${ }^{2}$ E-mail:1143960578@qq.com
}

\begin{abstract}
With the improvement of people's living standard, mass customization production model has been the trend of the furniture industry. A model of furniture product design and assembly for customization is proposed. It can decompose customers' needs and transform them into homologous modules set in the structure tree. Then through the assembly relationship graph and matrix of these product parts, an assembly sequence which can guide the assembly of products will be built by a genetic algorithm. This process will not only save manpower and resources, but also improve enterprise efficiency. In this article, an instance was given in order to verify the correctness of product design and assembly processes based on product family of mass customization.
\end{abstract}

Keywords: mass customization, assembly sequence, function-structure

\section{Introduction}

As customer needs change faster and faster, lags in the traditional mode of production make furniture companies cannot be good to keep up with changes of customer demands. Time, as an important factor, is raised to the primacy of production. Developing a new product model is crucial for furniture companies to make success in the increasingly fierce market competition.

Product family is the precondition for the implementation of mass customization and the core of mass customization technology. Product family is the innovation of technology from a single product to series products, containing all similar function and structure module. Most of the design can be completed with product family for different needs of different users. Product family is based on the normalized and standardized module which can provide a flexible, modular product model, conditions for variant design and agile design. Product family based on product platform provides a framework of product design. To the customers' demand for personalized, it can be solved by adding different personalization modules to provide rapid product design.

Furniture design model based on mass customization is intended to provide personalized products and services meeting customer demands using the cost and time of mass production [1]. This model can significantly improve the design efficiency, versatility and interchangeability of furniture and accessories, achieving the purpose of optimization design. A collection of product parts will be created by the production configuration model based on function-structure mapping, and through genetic algorithm will be generated by an assembly sequence. This process will meet the needs of individual customers to produce differentiated products that enable companies to have a lasting competitive advantage of differentiation. 
Now if a TV cabinet is customized by customer, it's function as follow: modern style, bamboo texture, both sides are cabinets with visible doors, easy to open and close door, middle of the cabinet divided into two layers, the lower is drawer. This paper will present the method with customized design and assembly process of the TV cabinet.

\section{Build Product Model based on Function-structure Mapping}

In the theory of mass customization, product platform and product family architecture are constituted of functional view, technical view and structural view. Functional view describes the functional requirements and its decomposition of product family; technical view describes the technical solutions to satisfy the functional requirements; structural view describes the physical structure of product family. There is a close relationship between the three views; the mapping relationship from the function view to the technical view referred to as product design; the mapping relationship from the technical view to structure view referred to as process design. The three views above are connected with each other and inseparable. American scholar proposed axiomatic design theory, the main concepts of axiomatic design theory including domain, layer, zigzag mapping and axiomatic design. Especially domain which is the most important concept of axiomatic design theory, design field is divided into four domains: customer domain, functional domain, physical domain and process domain. The emphasis of customer domain are customers, and the content is the customer's variety of different requirements to the product, which is the expectations of the product's features; the content of the designed product's functional domains is product features, that is, the products should have a series of functional which meet requirements; what make the physical domain description design parameters to meet the functional requirements and technical solutions, is the product function to instantiate the parameters by reference; process domain is used to describe the variables set that is needed in the process of product's manufacturing technology and process [10]. The relationship between these domains is sequence mapping. From theories above, a product configuration model is created based on mapping from the functional domain to the structural domain.

\subsection{Build Functional Free}

The object of functional domain describes the composition of the products and the relationship between them. Components are re-formed as a module on the basis of the analysis which includes functions and structures under specific circumstances. Users to customize and select the TV cabinet are usually considered in three areas: external appearance, placement and convenience. So TV cabinet features are divided into three subfunctions: appearance, placement and convenience. Appearance includes style and vein; placement includes basic placement function, horizontal separation and vertical separation; convenience includes movable space, grip, support, touch ground, close and other subfunctions, as shown as Figure 1, left.

\subsection{Build Structural Tree}

Structural tree describes the assembly hierarchy of product family and structure of all possible modules. According to the function-structure mapping principle above, the subfunctions of TV cabinet, such as appearance, placement and convenience, are mapped into decoration, main structure and accessory [2]. Main structure can be broken down into frame, clapboard and vertical board, respectively, to achieve the functions of basic placement, horizontal and vertical separation. Accessory can be subdivided into a drawer, handle, cabinet 
leg, kick plate and glass door panels and other modules, to achieve the corresponding subfunctions of the functional tree, as shown in Figure 1, right.

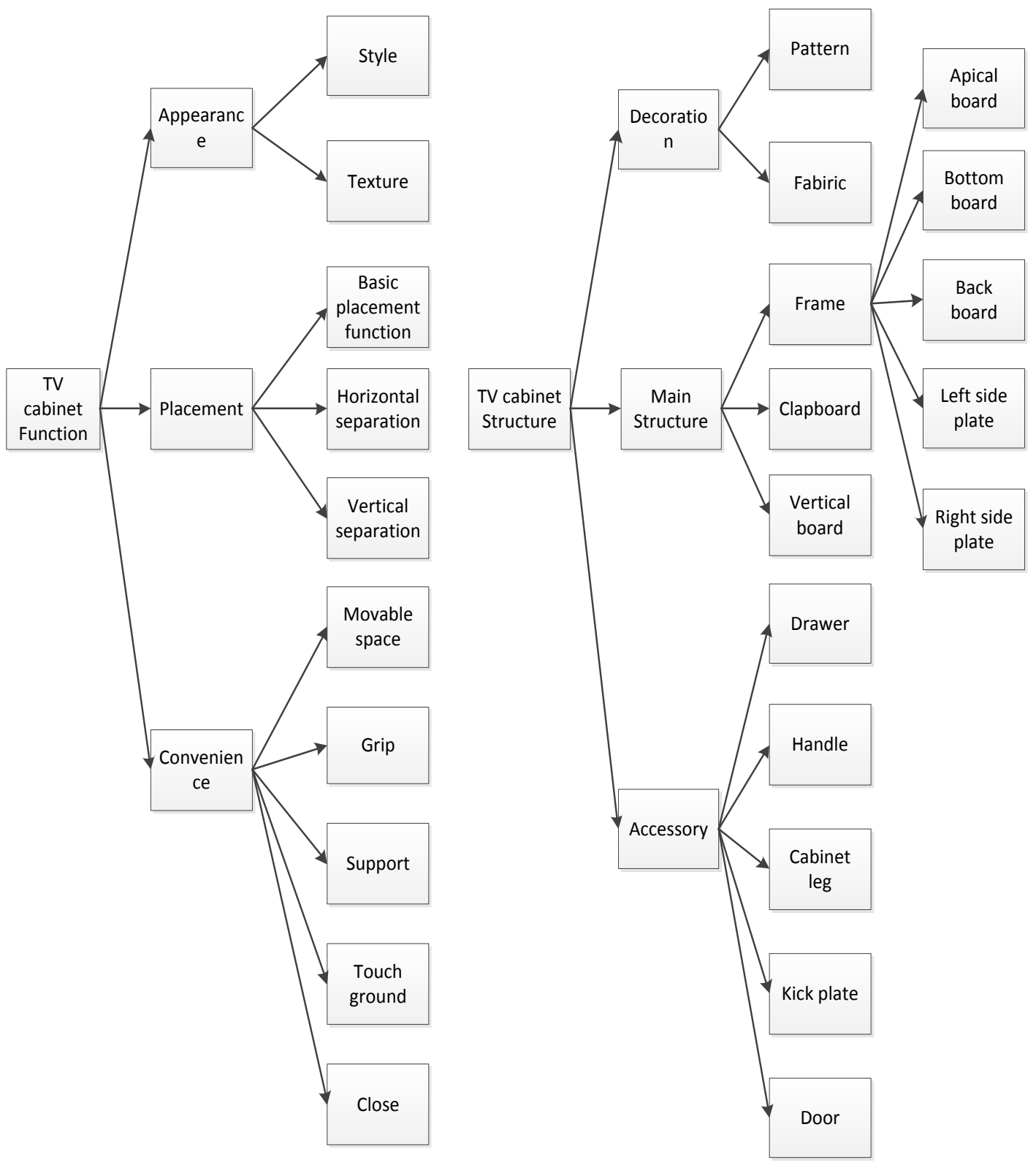

Figure 1. Functional Tree and Structural Tree of TV Cabinet

\subsection{Function-structure Mapping Principle}

According to set theory, when functional domain and structural domain have a mapping from functional expression space to structural expression space, it is demonstrated that two spaces have some function relation. Functions within the functional domain are decomposed into sub functions to make up a functional tree. These sub functions can be mapped into different structural units which have their specific functions of the product. The structural units can be used as a modular, need to be considered in function, structure and other sides. 


\subsection{Generalized Module Matrix Planning for Product Family}

According to the modular design principle, the product design transforms user's needs into product functional model. With the decomposition of the functional tree, the total product FR (Functional Requirement, FR) can be made up of multiple functional modules called $\mathrm{FR}_{\mathrm{i}}$ :

$\mathrm{FR}=\left[\mathrm{FR}_{1}, \mathrm{FR}_{2}, \ldots, \mathrm{FR}_{\mathrm{i}}, \cdots,{ } \mathrm{FR}_{\mathrm{k}}\right],(\mathrm{i} \in \mathrm{N})$

In Equation above: $\mathrm{FR}_{\mathrm{i}} \rightarrow \mathrm{PC}_{\mathrm{i}}$

PC (Product Component, PC) is the set of generalized structural modules; each functional module can be mapped to multiple generalized structural modules. According to product modular matrix planning method, generalized modular product family planning matrix is shown in Figure 2. Each column, there is a family of model-based products which main parameter changes from small to large. Each row, modules make up a product module chain [3].

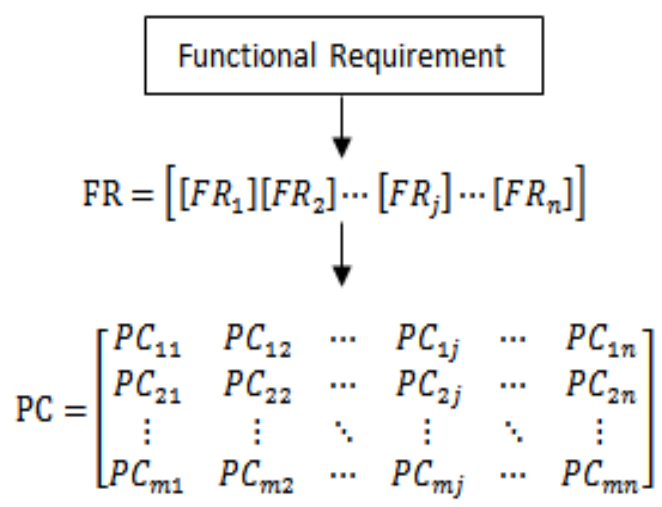

Figure 2. Product Family Oriented Generalized Module Matrix

\section{Functional Decomposition and Define the Structural Modules}

After obtaining users' demands, the system analyzes the total function of the product and decomposes it into sub-layer functions. Through the functional tree of TV cabinet, the customer's needs are finally decomposed as follow:

$\mathrm{FR}=[[[$ Modern Style $][$ Bamboo Texture $]][[$ Basic Placement Function] $[$ Horizontal Separation $\times 2][$ Horizontal Separation]][[Moveable Space][Grip][Support][Close]]]

An assembly components collection is mapped from the function-structure mapping model. According to the set of sub-function above, the TV cabinet's assembly components collection is $\mathrm{PC}=[\mathrm{PC} 1, \mathrm{PC} 2, \mathrm{PC} 3], \mathrm{PC} 1$ is decoration modules, $\mathrm{PC} 2$ is pattern modules, and PC 3 is accessory modules. PC1 and PC 3 will be added after the completion of PC2. The set of PC2 is shown as follow:

$\mathrm{PC} 1=$ [Modern style pattern, Bamboo fabric]

PC2 $=[[$ apical board, bottom board, back board, left side plate, right side plate][left vertical board, right vertical board, clapboard]]

PC $3=[$ drawer, handles $\times 2$, cabinet leg $\times 2$, glass doors $]$

In PC2, the side plates and vertical boards are the same height.

\section{Build Assembly Relationship Graph and Assembly Relationship Matrix}

An assembly relationship graph can be drawn based on the relationship between the components of PC2 [4]. It is an undirected graph, forming with nodes and edges of connected 
nodes, in which nodes represent components and edges mean the correlation between these components, which is the assembly relationship between two different components. Through the exploded view of assembly and analysis of the relationship between the assembly parts, a graph can be drawn. According to the assembly relationship of the parts in PC2, the TV cabinet's assembly relationship graph is shown in Figure 3.

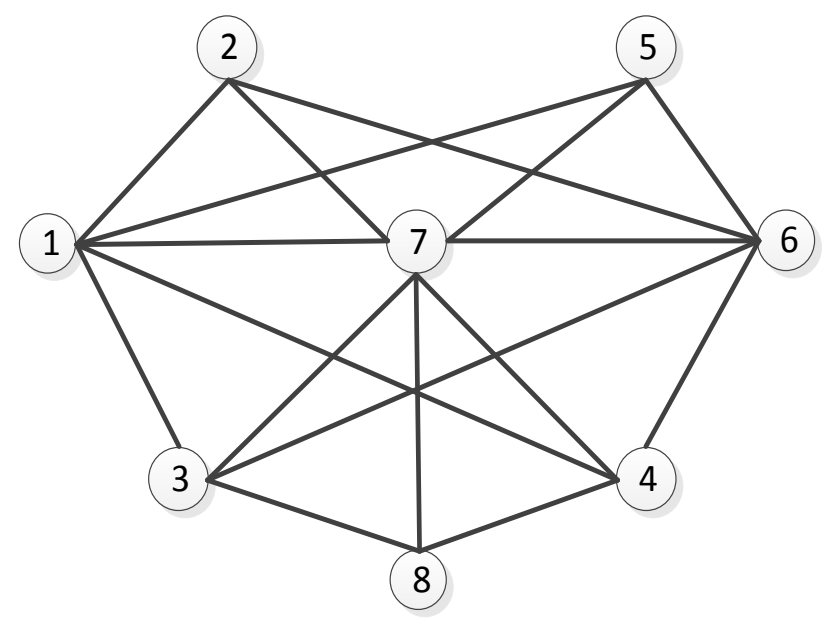

Figure 3. TV Cabinet's Assembly Relationship Graph.

In Figure 3, number 1-8 represents apical board, left side plate, left vertical board, right vertical board, right side plate, bottom board, back board and clapboard. According to the assembly relationship graph above, a matrix of $\mathrm{N}$ rows and $\mathrm{N}$ columns expresses the relationship of assembly process can be created, shown as follow matrix:

$$
\mathrm{R}_{\mathrm{n} \cdot \mathrm{n}}=\left[\begin{array}{ccc}
r_{11} & \ldots & r_{1 \mathrm{n}} \\
\vdots & r_{\mathrm{ii}} & \vdots \\
r_{\mathrm{n} 1} & \ldots & r_{\mathrm{nn}}
\end{array}\right]
$$

In this matrix, $r_{i, j}=1$, shows there is a related surface or edge between node $i$ and $j$, otherwise, $r_{i, j}=0$, shows that there is no related surface or edge between node $i$ and $j$. If $i=j$, , namely the same node, in order to facilitate the calculation, that it is completely unrelated, and its value is 0 . It can be seen that this matrix is a symmetric matrix. According to the TV cabinet assembly relationship graph, an assembly relationship matrix in which 8 rows and 8 columns shown as Figure 4:

$$
R_{7-7}=\begin{array}{lllllllll}
1 & 1 \\
2 \\
3 \\
4 \\
5 \\
6 \\
7 \\
8
\end{array}\left[\begin{array}{lllllllll}
0 & 1 & 3 & 4 & 5 & 6 & 7 & 8 \\
1 & 0 & 0 & 0 & 0 & 1 & 1 & 0 \\
1 & 0 & 0 & 0 & 0 & 1 & 1 & 1 \\
1 & 0 & 0 & 0 & 0 & 1 & 1 & 1 \\
1 & 0 & 0 & 0 & 0 & 1 & 1 & 0 \\
0 & 1 & 1 & 1 & 1 & 0 & 1 & 0 \\
1 & 1 & 1 & 1 & 1 & 1 & 0 & 1 \\
0 & 0 & 1 & 1 & 0 & 0 & 1 & 0
\end{array}\right]
$$

Figure 4. Assembly Relationship Matrix of TV Cabinet 
Assembly relationship matrix can be better equipped to express the product assembly relationship information. Using assembly relationship matrix and genetic algorithm, an assembly sequence is created which can guide the actual assembly process.

\section{Generation of Assembly Sequence}

Based on the assembly relationship graph and matrix above, an assembly sequence guiding assembly process can be created by genetic algorithm.

\subsection{Establishment of Adaptive Function}

To establish the objective function is mainly in three areas as the primary measure, the number of assembly direction, stability and geometric feasibility [5].

Number of assembly direction: In assembly process, the fewer of the assemble direction number of a part, the more difficult rearward assembly, and the assemble difficulty is increasing as geometric progression with the reducing of the assemble direction number of the part [6-8]. Therefore, the parts with less assemble direction number should be placed in the front of the assembly sequence. For each part, the assemble direction number called y is defined as following formula (1):

$$
f_{1}=\left\{\begin{array}{cc}
0 & 1 \leq y \leq 2 \\
0.2 \times n & 2<y \leq 4 \\
1 & y \geq 5
\end{array}\right.
$$

Stability of the assemblage: Through assembly relationship matrix, the relationship of this assemblage then accumulates the connection of the adjacent parts in assembly sequence. Between two components, if there is a connection then plus 1 , if there is not then plus 0 . That is plus corresponding value in matrix. V stands for the value obtained above; and obviously, $0 \leqslant \mathrm{~V} \leqslant 2 \mathrm{~N}-2, \mathrm{~N}$ is the total number of parts in assembly sequence. The following formula (2) can evaluate the stability of the assembly process:

$$
f_{2}=\frac{2 N-2-V}{2 N-2}
$$

Geometric feasibility: The geometric feasibility refers the removable nature of the assembly sequence. It can be evaluated by this formula $f_{3}=(N(N-1)) / 2-M ; M$ is the interference number occurred in the sequence $A i$.

Adaptive function: Through analysis of assemble direction number, stability of assemblage and geometric feasibility, the adaptive function can be calculated by equation: $\mathrm{F}=\omega_{1} f_{1}+\omega_{2} f_{2}+\omega_{3} f_{3} ; \omega_{i}$ represents the weight of $f_{i}$

\subsection{Generate Assembly Sequences using Genetic Algorithm}

After Step 1, the next options are coding chromosome, generation of the initial population, mutation, crossover operator and selection, etc. Evolved over several generations, the product assembly sequence is: $7,1,3,4,6,8,5,2$.

\subsection{Assemble Other Accessories}

Assembled decorative modules of PC1 and accessories of PC3 to the assemblage produced through the steps above, the TV cabinet is finished. 


\section{Conclusion}

Through the product model of function-structure mapping, layers of functional modules are decomposed from users' needs. They are transformed into corresponding modules in structural tree and generated to an assembly sequence by the genetic algorithm. This process combines the design and assembly process together so as to save manpower and resources. At the same time, improve enterprise manufacturer efficiency and reduce the production cycle of the product. Thus enhance the enterprise's competitive power and expand the enterprise's economic benefits. This paper presented this method by customizing a TV cabinet. Such method provides the basis for a wide range of technology and research applications. However, this article did not involve the technology areas, not able to intuitively understand the product's design in the process of design and assembly. So in the process of building product family model, much further researches still to be done.

\section{Acknowledgements}

This work is supported by the Fundamental Research Funds for the Central Universities, China, DL12BB08.

\section{References}

[1] R. B. Stone, "Using quantitative functional models to develop product architecture", Design Studies, vol. 21, (2000), pp. 239-260.

[2] M. Li, "Study on the Modular Design of Integrated Cupboard", (2009).

[3] Y. Hu and Y. Xu, "Product Dynamic Family Model Oriented on Generalized Modular Design/Manufacture", Machine Tool \& Hydraulics, vol. 11, (2006), pp. 24-27.

[4] X. Han and W. Chang, "A new assembly sequence planning method based on the genetic algorithm", Machinery, vol. 35, (2008), pp. 56-60.

[5] T. Lihra and U. Buehlmann, "Consumer perception of mass customized furniture", Proceedings of the International Scientific Conference on Hardwood Processing (ISCHP 2009), (2009) October 10-13, Shanghai, China.

[6] E. T. Kodzi Jr., "Mass customization as a framework for manufacturing transformations in the United States wooden furniture industry", Purdue University, (2006).

[7] J. Daaboul, C. Da Cunha, A. Bernard and F. Laroche, "Design for mass customization: Product variety vs. process variety", CIRP Annals-Manufacturing Technology, vol. 60, (2011), pp. 169-174.

[8] R. F. LU, "Modeling of Customer Decision Point and Design Change Impact in Customized Large Manufacturing System Integration”, Proceedings of the 3rd Interdisciplinary World Congress on Mass Customization and Personalization (MCPC 2005), (2005), Hong Kong, China.

[9] D. Kofjač and M. Kljajic, "Furniture Production Optimization with Visual Simulation and Genetic Algorithms", 8th WSEAS International Conference on simulation, modeling and optimization (SMO '08), (2008), pp. 53-58.

[10] P. R. Dean, D. Xue and Y. L.Tu, "Prediction of manufacturing resource requirements from customer demands in mass-customisation production", International Journal of Production Research, vol. 47, (2009), pp.1245-1268.

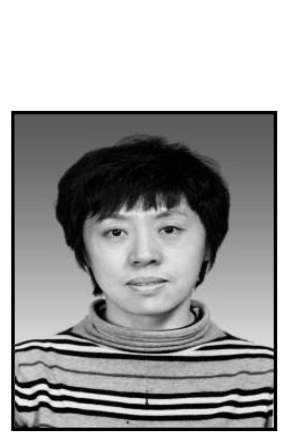

\section{Author}

Qi Yue, College of Information and Computer Engineering, Northeast Forestry University, China. She was born in March 1968, Research direction: Artificial Intelligence and Intelligent Control, Computer Aided Manufacturing. 
International Journal of Smart Home

Vol. 9, No. 3 (2015) 